\title{
Artificial Swimmers Propelled by Acoustically Activated Flagella
}

\section{Journal Article}

Author(s):

Ahmed, Daniel; Baasch, Thierry; Jang, Bumjin; Pané, Salvador; Dual, Jürg; Nelson, Bradley (D)

Publication date:

2016-08-10

Permanent link:

https://doi.org/10.3929/ethz-b-000118859

Rights / license:

In Copyright - Non-Commercial Use Permitted

Originally published in:

Nano Letters 16(8), https://doi.org/10.1021/acs.nanolett.6b01601 


\section{Artificial swimmers propelled by acoustically}

\section{activated flagella}

Daniel Ahmed ${ }^{1}$, Thierry Baasch ${ }^{2}$,Bumjin Jang ${ }^{1}$, Salvador Pane $^{1}$, Jürg Dual $^{2}$ and Bradley J. Nelson*1

${ }^{1}$ Institute of Robotics and Intelligent Systems (IRIS), ETH Zurich, Zurich, CH-8092, Switzerland

${ }^{2}$ Institute of Mechanical Systems (IMES), ETH Zurich, Zurich, CH-8092, Switzerland ${ }^{*}$ Correspondence and requests for materials should be addressed to B.J.N (e-mail: bnelson@ethz.ch, Tel: +41 4463255 29) 


\begin{abstract}
Recent studies have garnered considerable interest in the field of propulsion to maneuver micro and nano-sized objects. Acoustics provide an alternate and attractive method to generate propulsion. To date, most acoustic-based swimmers do not use structural resonances, and their motion is determined by a combination of bulk acoustic streaming and a standing-wave field. The resultant field is intrinsically dependent on the boundaries of their resonating chambers. Though acoustic based propulsion is appealing in biological contexts, existing swimmers are less efficient, especially when operating in vivo, since no predictable standing-wave can be established in a human body. Here we describe a new class of nanoswimmer propelled by the small-amplitude oscillation of a flagellum-like flexible tail in standing and, more importantly, in traveling acoustic waves. The artificial nanoswimmer, fabricated by multistep electrodeposition techniques, compromises a rigid bimetallic head and a flexible tail. During acoustic excitation of the nanoswimmer the tail structure oscillates, which leads to a large amplitude propulsion in traveling waves. FEM simulation results show that the structural resonances lead to high propulsive forces.
\end{abstract}

KEYWORDS: acoustic, ultrasound, propulsion, nanoswimmer, nanomotor, flagella 
The physics of motion in liquid at micro-and nanoscales, where viscous forces dominate over inertial forces, is distinct from inertia-dominated counterparts ${ }^{1}$. Realizing propulsion of micro and nano sized objects is challenging, however, systems capable of precise motion could enable critical applications in targeted therapeutics ${ }^{2-6}$, non-invasive surgery $^{7}$, material assembly ${ }^{8}$, lithography ${ }^{9}$, and water treatment ${ }^{10-14}$. To date, there exist numerous artificial swimmers fabricated at different length scales ${ }^{15}$, which are propelled utilizing different physical and chemical principles. Autonomous motion is achieved by converting energy from the environment to generate motion ${ }^{16}$. For example, chemical energy is harvested by bimetallic motors that move by self-electrophoresis, decomposing fuel asymmetrically over their surfaces ${ }^{17-19}$. Motion can also be modulated by chemical or optical gradients ${ }^{20}$, local analyte concentrations ${ }^{21}$, electrochemical control, ${ }^{22}$ or by periodic contraction of cardiomyocytes on engineered surfaces ${ }^{23,24}$. Propulsion can also be derived by exploiting external fields such as magnetic ${ }^{25-33}$, electric ${ }^{34}, \operatorname{light}^{35}$, thermal, ${ }^{36,37}$ and acoustics $^{32,38-43}$. Acoustically propelled micro and nanoswimmers are compelling in biological or biomedical contexts since many of the existing swimmers that use electric fields can not be used in biological environments such as the human body. Recent studies utilized acoustics to demonstrate propulsion by oscillating bubbles trapped within soft micro-containers ${ }^{40}$ and by scattering of acoustic waves in non-resonating bimetallic nanowires ${ }^{39}$. The latter was able to observe the motion of the micro-rods in a resonating cylindrical chamber. However, the propulsion mechanism was not fully explained, and it remains to be seen if the motion is autonomous or due to external effects such as acoustic streaming ${ }^{39}$ within the chamber. Developing traveling-wave induced swimmers will become necessary for next generation artificial micro/nano-swimmers, as they will enable in vivo applications. Here, we demonstrate a new class of nanoswimmer that moves through an aqueous solution by small-amplitude oscillation of a flagellum-like polypyrrole tail in the 
presence of acoustic waves. These nanoswimmers respond to ambient acoustic energy and can propel in standing and, more importantly, in traveling acoustic waves.

Our nanoswimmer consists of two parts, an acoustically activated flagellum, i.e. the tail, and a bimetallic head, as shown in Fig. 1a. In the presence of an acoustic field, the nanoswimmer is excited to an asymmetric bending resonance mode. Each nanoswimmer is fabricated by a multistep electrodeposition technique ${ }^{30,44}$ (see also Supporting Information). The thin elastic rod-like flagellum is made of polypyrrole (PPy), while the head is made of a nickel and gold (Ni/Au) composite. Acoustic waves are used to excite the swimmer flagellum, which contributes to propulsion. We exploit this phenomenon to achieve self-propulsion.

We studied nanoswimmer propulsion in both standing and traveling acoustic waves. A complex standing-wave field is formed by introducing acoustic waves via a piezoelectric transducer bonded on a glass slide adjacent to the liquid droplet. The droplet is then sandwiched between glass slides (see Supplementary Fig. 1 for the experimental details). Node formation, which is a characteristic of a standing wave field, is verified by trapping $\sim 10 \mu \mathrm{m}$ polystyrene microparticles. The complex pattern formation is similar to Chladni's plate vibration ${ }^{45-47}$ (see Supplementary Fig. 2). To generate traveling acoustic waves in the setup, we designed a Polydimethylsiloxane (PDMS)-based acoustic chamber bonded on a glass slide adjacent to the piezoelectric transducer, as demonstrated in Fig. 1b. PDMS is used as the chamber because the acoustic impedance mismatch between water and PDMS is similar, and $97 \%$ of the waves are transmitted away from the microchannel ${ }^{48}$. The total thickness of the PDMS surface is designed to be $\sim 1 \mathrm{~cm}$, much thicker than the channel height $h \sim 80-100 \mu \mathrm{m}$. The thickness ensures dampening of acoustic waves during propagation across the lossy PDMS medium due to attenuation. This eliminates the possible 
formation of nodal points/contours due to the weak reflection of waves from PDMS boundaries and provides a predominant traveling acoustic field.

We analyzed the propulsion characteristics in a standing wave field first. Once the nanoswimmer submerged in the liquid is exposed to an acoustic field with a wavelength much larger than the swimmer dimension, the PPy-based flagellum begins to oscillate. The resulting oscillation induces a steady local streaming flow field, which is characterized by a pair of counter-rotating vortices at a length scale comparable to the size of the swimmer, as demonstrated in Fig. 1c. The micro/nanostreaming flow field near the nanoswimmer can be modeled via a perturbation expansion approach, considering only small density variations of the acoustic wave. The oscillatory first-order fields induce a second-order steady flow (also known as the steady acoustic microstreaming) which is governed by a Stokes equation driven by a Reynolds stress. This Reynolds stress is the driving force behind the steady local acoustic microstreaming. When the frequency of the function generator driving the transducer approaches the resonance frequency of the tail, the vibrational amplitude of the tail becomes significant enough to induce motion. The transducer has a first resonance at $2.8 \mathrm{KHz}$ and multiple higher resonances. The swimmer shows propulsion at around $90 \mathrm{kHz}$, which can be attributed to an overlap of swimmer and transducer resonance, where the transducer resonances lead to high acoustic energy in the system but the swimmer resonance is the main propulsion mechanism and leads to high velocity swimming motion compared to other nonresonating particles. Fig. 2a demonstrates translational locomotion of a nanoswimmer moving left to right (see also supplementary video 2 ). To gain insight into the acoustic field, polystyrene particles are introduced into the liquid as tracers. Not surprisingly, some particles move. However, the nanoswimmer propels with a velocity distinctly higher than the mean velocity of the particles, as demonstrated in Fig. 2b. We believe that the motion of the tracers comes forth due to acoustic Rayleigh (bulk) streaming ${ }^{49,50}$ within the chamber 
i.e. millimeter-to -centimeter scale circulation within the liquid due to attenuation of the vibration within a thin boundary layer on the glass slide. (For the sake of simplicity, this is referred to as external streaming). Fig 2 shows the swimmer drifts, which can be attributed to this external streaming.

The force created by the acoustically induced tail was tuned by adjusting the intensity of the applied acoustic field i.e. the voltage applied to the piezoelectric transducer (the higher the voltage, the larger the force). It is shown that a linear relationship exists between the voltage $V_{0}$ applied to the piezo transducer and the acoustic pressure $P_{0}$ by $V_{0} \propto P_{0},{ }^{40,51-54}$ with the input signal frequency held constant. The propulsive force is, therefore, proportional to the square of the applied acoustic pressure or the input voltage, $F_{A} \propto P_{0}^{2} \propto V_{0}^{2}$ (acoustic propulsive force is discussed later). As the swimmer moves through the liquid, the drag force is developed. The Reynolds number of the swimmer is estimated to be lower than 0.0015 . At low Reynolds number, the drag force is linearly proportional to the swimmer velocity, i.e. $F_{D} \propto v_{S}$. Experiments indicate that as the input signal frequency approaches the resonant frequency of the tail, the swimmer begins to move. It very quickly reaches terminal velocity, at which $F_{A}=$ $F_{D}$. Therefore, the velocity of the nanoswimmer can be controlled via the input voltage through the relation $v_{s} \propto V_{0}^{2}$. This relation was demonstrated empirically with nanoswimmers traveling through water, as shown in Fig. 3a. The data shows that the quadratic relation agrees reasonably well with theory $\left(v_{s} \propto V_{0}^{2.5}\right)$. The small deviation from a precise quadratic relation can be due to complex standing-wave fields and bulk streaming flow patterns established in the chamber $^{55,56}$.

We analyzed the frequency response of a 15 to $20 \mu \mathrm{m}$ in length and 0.3 to $0.6 \mu \mathrm{m}$ in diameter swimmer. The excitation frequency is modulated from 20 to $100 \mathrm{kHz}$, while maintaining the applied voltage constant at $10 \mathrm{~V}_{\mathrm{PP}}$ (maximum voltage applied under the 
current experimental setup). The piezo transducer (KPEG-126, Kingstate) has the lowest resonance frequency at $2.8 \mathrm{KHz}$. The piezo transducer contains multiple resonances above the first resonance. We believe that the swimmer is excited at frequencies where both the transducer and the swimmer are at resonance. No significant propulsion was observed except when the excitation frequency approaches $90.0 \mathrm{kHz}$. The resonance response indicated by swimmer velocity is shown in Fig. 3b, with velocity peaks at around $91.5 \mathrm{kHz}$. The resonance is reasonably sharp and, for typical excitation amplitudes, a swimmer at rest will move when excited near resonance.

We then studied contributions, if any, of each of the disassembled entities of the nanoswimmer. Control experiments were established with individual gold, nickel, and goldnickel nanowires, respectively, for a frequency sweep between $89-93 \mathrm{kHz}$. No discernable propulsion was observed for copper nanowires. Propulsion velocities under $10 \mu \mathrm{m} / \mathrm{s}$ were found for gold and gold-nickel counterparts, however, almost an order magnitude higher propulsion velocity is attained when a flexible appendage, $i$. e. a flagellum, is attached to the bimetallic head, as demonstrated in Fig. 3b. The resonances of gold and nickel entities are in the $\mathrm{MHz}$ range, approximately two orders higher than the estimated resonance of the PPy tail. This shows that the metal structures are not resonating at the excitation frequency range. The small peaks of the metallic structures in Fig. $\mathbf{3 b}$ are probably due to the transducer resonance system since the resonances of the metallic structures are in the high $\mathrm{MHz}$ range (see also Supplementary Fig. 3). We can infer that the slight motion of these dissembled entities could be due to external streaming of the rather complex field established in the setup. Ideally, all the peaks should have been at similar frequencies, however, since experiments were done on different chips and transducers, there is a small shift in the resonance peaks. 
To understand the propulsion mechanism better, while reducing the complexities in the field, we then tested our nanoswimmer in predominant traveling acoustic waves (see Fig. 1b and experimental details). The setup ensures that multiple acoustic phenomena such as the standing wave formation and the bulk (external) streaming are eliminated or reduced. Swimmers along with polystyrene particles $(\sim 10 \mu \mathrm{m})$ were introduced into the microchannel. The excitation frequency of the tail, as expected should remain the same whether the swimmer is exposed to standing or traveling acoustic waves. When the excitation frequency approaches $\sim 91 \mathrm{kHz}$, the nanoswimmer undergoes translational motion as demonstrated in Fig. 4. The swimmer contains multiple tails and the swimmer does not rotate along its major axis. Multiple tails are evident when the acoustic is turned off (see Supplementary Video 4). Since polystyrene particles of diameter $\sim 10 \mu \mathrm{m}$ remained stationary during propulsion, we conclude that no standing wave field is formed. However, large stationary particles do not confirm the absence of external streaming, which could also lead to motion of the nanoswimmer. Consequently, experiments were carried out with swimmers dispersed in $\sim 0.5 \mu$ m polystyrene particles. Fig. 5 captures the translation motion of the nanoswimmer moving left to right while the particles undergo random Brownianmotion suggesting the absence of external streaming. In addition, the swimmers move in straight lines in travelling acoustic waves, which may also suggest that the external streaming is minimum in the chamber. The lack of external streaming is also confirmed within the chamber by the immobilized severed bimetallic head, marked by the blue arrow in Fig. 4a. This confirms that a flexible PPy tail, i.e. a flagellum, is necessary for efficient propulsion in traveling acoustic waves. We studied propulsion in predominant travelling acoustic waves by injecting the swimmers into the chamber. We have not characterized at which plane the swimmer propels, but at different time-scale, the swimmer sediments to different planes. We 
have experimentally found that the swimmers propel, irrespective of different planes, in the presence of ultrasound.

The driving mechanism behind the motion of the swimmer is the force due to linear and nonlinear interactions of the swimmer with the acoustic field, referred to as the propulsive force. As soon as the swimmer is driven at resonance, the propulsive force increases in magnitude, and the swimmer starts to move. The link between high amplitudes of the propulsive force and structural resonances of the swimmer can be modeled by FEM simulation using COMSOL Multiphysics. This shows that structural resonance ${ }^{57}$ can lead to maximal propulsive forces, which allows for strong autonomous motion even in the traveling wave situation.

In order to resolve the acoustic fields and the microstreaming motion associated with the nanoswimmer, we used a perturbation approach, since a direct Navier-Stokes simulation would require tremendous computational power. We simulated in $2 \mathrm{D}$ because $3 \mathrm{D}$ simulation requires an enormous number of finite elements and is not possible with reasonable computational power. The local acoustic microstreaming response of the fluid by the nanoswimmer can be characterized by the second-order system of equations $\left(v_{2}, p_{2}, \rho_{2}\right)$, which in turn is driven by the time-harmonic $\left(e^{-i \omega t}\right)$ first-order fields $\left(\mathrm{v}_{1}, \mathrm{p}_{1}, \rho_{1}\right)$. The fluid response is governed by the standard Navier-Stokes equations for a linear, viscous compressible fluid:

$$
\begin{gathered}
\frac{\partial \rho}{\partial \mathrm{t}}+\nabla \cdot(\rho \mathbf{v})=0 \\
\rho \frac{\partial \mathbf{v}}{\partial \mathrm{t}}+\rho(\mathbf{v} \cdot \nabla) \mathbf{v}=-\nabla \mathrm{p}+\mu \nabla^{2} \mathbf{v}+\left(\mu_{\mathrm{b}}+\frac{1}{3} \mu\right) \nabla(\nabla \cdot \mathbf{v})
\end{gathered}
$$

where $\rho$ is the mass density of the fluid, $\mathrm{p}$ is the fluid pressure, and $\mu$ and $\mu_{\mathrm{b}}$ are the shear and the bulk dynamic viscosities, respectively. We employ Nyborg's perturbation approach where the fluid velocity, density, and pressure are assumed to be of the following form: 


$$
\begin{gathered}
\mathrm{v}=\mathrm{v}_{1}+\mathrm{v}_{2}+\mathrm{O}\left(\varepsilon^{3}\right)+\cdots, \\
\mathrm{p}=\mathrm{p}_{0}+\mathrm{p}_{1}+\mathrm{p}_{2}+\mathrm{O}\left(\varepsilon^{3}\right)+\cdots, \\
\rho=\rho_{0}+\rho_{1}+\rho_{2}+O\left(\varepsilon^{3}\right)+\cdots .
\end{gathered}
$$

where $\varepsilon$ is a non-dimensional parameter and is defined as $\frac{\left|\rho_{1}\right|}{\left|\rho_{0}\right|}=\varepsilon \ll 1$. Substitution of (3) in (1) and (2), and segregation of first-order terms yields a first-order system:

$$
\begin{gathered}
\frac{\partial \rho_{1}}{\partial \mathrm{t}}+\rho_{0}\left(\nabla \cdot \mathbf{v}_{1}\right)=0 \\
\rho_{0} \frac{\partial \mathbf{v}_{1}}{\partial \mathrm{t}}=-\nabla \mathrm{p}_{1}+\mu \nabla^{2} \mathbf{v}_{1}+\left(\mu_{\mathrm{b}}+\frac{1}{3} \mu\right) \nabla\left(\nabla \cdot \mathbf{v}_{1}\right)
\end{gathered}
$$

The first order equations are fully coupled with the structural mechanics of the swimmer. This is achieved by coupling the first order acoustic equations with the structural mechanics of the swimmer in COMSOL. A subsequent time-averaging over an oscillation period yields the second-order system of equations:

$$
\begin{aligned}
& \left\langle\frac{\partial \rho_{2}}{\partial \mathrm{t}}\right\rangle+\rho_{0} \nabla \cdot\left\langle\mathbf{v}_{2}\right\rangle=-\nabla \cdot\left\langle\rho_{1} \mathbf{v}_{1}\right\rangle \\
& \rho_{0}\left\langle\frac{\partial \mathbf{v}_{2}}{\partial \mathrm{t}}\right\rangle+\left\langle\rho_{1} \frac{\partial \mathbf{v}_{1}}{\partial \mathrm{t}}\right\rangle+\rho_{0}\left\langle\left(\mathbf{v}_{\mathbf{1}} \cdot \nabla\right) \mathbf{v}_{1}\right\rangle=-\nabla \mathrm{p}_{2}+\mu \nabla^{2} \mathbf{v}_{2}+\left(\mu_{\mathrm{b}}+\frac{1}{3} \mu\right) \nabla\left(\nabla \cdot \mathbf{v}_{2}\right)
\end{aligned}
$$

The vibrating first order motion of the swimmer leads to a time averaged second order velocity on its boundary. At the boundaries of the swimmer, the Stokes drift velocity condition was applied which is given by $\mathrm{v}_{\mathrm{S}}=\frac{i}{\omega}\left\langle\mathrm{v}_{1} \cdot \nabla \mathrm{v}_{1}\right\rangle=-\mathrm{v}_{b}$ (if an $e^{-i \omega t}$ ansatz is used in the acoustical domain). This takes into account the time averaged first order velocity of the vibrating boundary.

We successively solved the first and the second-order equations successively to obtain the first order and second order fields. The acoustic radiation force gives rise to the propulsive 
force acting on the swimmer and is composed by the surface integral of the stress $\left\langle\boldsymbol{\sigma}_{2}\right\rangle$ due to microstreaming and the nanoswimmer vibration $\mathbf{v}_{1}$, acting on the surface of the nanoswimmer, out to a surface $\partial \Omega_{1}$. Taking $\boldsymbol{n}$ to be outward normal pointing away from the swimmer, we obtain the total propulsive force $\mathrm{F}_{\mathrm{A}} \cdot{ }^{.58}$

$$
\mathrm{F}_{\mathrm{A}}=\oint_{\partial \Omega_{1}}\left\langle\boldsymbol{\sigma}_{2}\right\rangle \cdot \boldsymbol{n} d A+\oint_{\partial \Omega_{1}} \rho_{0}\left\langle\mathbf{v}_{1} \mathbf{v}_{1}\right\rangle \cdot \boldsymbol{n} d A
$$

The angle bracket $\langle\cdot\rangle$ represents time-averaging over one acoustic period. The first term in (8) is due to the stresses developed by localized acoustic microstreaming, and the second term arises from the structural vibration of the nanoswimmer. The first and the second excited modes of the nanoswimmer are shown in Fig. 6a. Fig. 6b shows the simulated acoustic radiation force and the time averaged elastic strain energy of the swimmer. It can be seen that maximal acoustic radiation forces coincide with maximal elastic strain energy, this leads to the conclusion that structural resonances play an important role in the propulsion mechanism. The numerical results suggest that the swimmer excited at the second harmonics will move in the direction of the tail. However, we have not yet been able to verify this experimentally. The simulation at the first harmonics, although yielding only qualitative results, shows a resulting force in the direction of the swimmer head as seen experimentally. We like to mention that the point of the numerical simulation is to show that the resonance of the swimmer can be excited by an external wave and leads to amplification of the acoustic forces. It was not possible to carry out the simulation at lower frequencies as the relation between the swimmer dimension and the wavelength becomes too extreme, which leads to an ill-conditioned numerical problem, hence we carried out the simulation around $(1 \mathrm{MHz})$. The acoustic microstreaming for the first and the second mode are shown in Fig. 6c and Fig. 6d, respectively in liquid water. Due to the ill conditioning of the numerical problem it was not possible to acquire precise force values for the first harmonic. The numerical resolution of the velocity gradients of the streaming at the 
first harmonic was not sufficiently stable to obtain quantitative values for the acoustic radiation force. However, it was possible by applying a lower numerical tolerance of $10^{-3}$ instead of $10^{-4}$ to calculate the qualitative streaming flow.

For a nanoswimmer oscillating at the fundamental frequency, the liquid flow patterns are characterized by a pair of symmetric vortices developed near the head and the tail. The simulated streaming results at the fundamental frequency, Fig. 6a, and matches well with the experimental streaming result, Fig. 1c. The streaming flow pattern is rather complicated and strongly depends on the excited frequency. The direction of the swimmer is sensitive to the excited frequency and its direction cannot be deduced from the streamlines of microstreaming only. Both acoustic microstreaming $\left(\mathbf{v}_{2}\right)$ and structural vibration $\left(\mathbf{v}_{1}\right)$ contribute to the swimming direction. It is not possible to deduce direction of the total propulsive force from the microstreaming part only. The resonance frequency of the nanoswimmer in water was experimentally determined to be $(\sim 90 \mathrm{kHz})$, whereas, the simulated value in water was found at $(\sim 240 \mathrm{kHz})$. Though the resonance frequencies (fundamental) are of similar order of magnitude, the discrepancies are reasonable since no uniform information regarding the Young's modulus for polypyrrole nanowire exist. In addition, since the simulation is performed in 2D, the swimmer behaves stiffer. A 3D simulation can contribute to a better estimation of the frequencies; however, performing $3 \mathrm{D}$ simulation is virtually impossible for the nanoswimmer because structural mechanics and viscous acoustics need to be resolved, which requires a tremendous number of mesh elements. Also, the discrepancies could be due to variations in length of each entity of the nanoswimmer arising from the fabrication.

The resonance frequency is extremely sensitive to the swimmer dimensions. Future work will include propulsion of various sizes and their corresponding eigenmodes, investigate the directionality of the swimmer and swimmer manipulation in acoustic and magnetic fields. 


\section{ASSOCIATED CONTENT}

\section{Supporting Information.}

Experimental procedures and movies regarding controls and propulsion of the nanoswimmer.

\section{Simulation (COMSOL) Setup}

Three different physical models have been used during the simulation of the microswimmer. Far away from the swimmer the fluid has been modeled as inviscid fluid and the outer boundaries have been modeled as perfectly matched layers, modeling an infinite domain. In the vicinity of the swimmer, the fluid has been modeled using the viscous first order equations from the perturbation expansion. The swimmer was modeled using the structural mechanics module and all the domains were fully coupled. The background wave field magnitude was chosen to be $(1 \mathrm{kPa})$, and the direction of the wave was orthogonal to the swimmer. In a second step, the results of the first order simulation were used to perform a streaming simulation.

\section{Dimension and material parameters of the nanoswimmer used in simulation.}

The Young's modulus of the polypyrrole was set to $(0.1 \mathrm{GPa})^{59}$, and the density was estimated as $1600 \mathrm{~kg} / \mathrm{m}^{3}$. The thickness was set to $0.6 \mu \mathrm{m}$, the length of the polypyrrole tail was set to $8.8 \mu \mathrm{m}$, the gold region was $1.8 \mu \mathrm{m}$ and the nickel head was $7.3 \mu \mathrm{m}$.

\section{AUTHOR INFORMATION}

\section{Corresponding Author}

*E-mail: bnelson@ethz.ch

\section{Author contributions}

D.A. conceived the idea. D.A. and T.B. contributed to the experimental design and scientific presentation. D.A. performed all the experiments and data analysis. T.B., D.A. and J.D. 
contributed to the theoretical analysis. S.P. and B.J. designed and fabricated the nanoswimmers. All authors contributed to the scientific discussion and writing of the manuscript. B.J.N. provided the overall guidance and contributed to the scientific presentation.

\section{Notes}

The authors declare no competing financial interests.

\section{ACKNOWLEDGEMENT}

Funding for this research was also provided by the European Research Council Advanced Grant "Microrobotics and Nanomedicine (BOTMED). S.P. acknowledges financial support by the European Research Council Starting Grant "Magnetoelectric Chemonanorobotics for Chemical and Biomedical Applications (ELECTROCHEMBOTS)", by the ERC grant agreement no. 336456. We also thank Ivo Leibacher and Mahmut Selman Sakar for helpful discussions. 


\section{REFERENCES}

(1) Purcell, E, M. Am. J. Phys. 1977, 45 (1), 3-11.

(2) Patra, D.; Sengupta, S.; Duan, W.; Zhang, H.; Pavlick, R.; Sen, A. Nanoscale 2013, 5 (4), 1273-1283.

(3) Walker, D.; Käsdorf, B. T.; Jeong, H.-H.; Lieleg, O.; Fischer, P. Sci. Adv. 2015, 1 (11), e1500501-e1500501.

(4) Medina-Sánchez, M.; Schwarz, L.; Meyer, A. K.; Hebenstreit, F.; Schmidt, O. G. Nano Lett.2015, 16 (1), 555-561.

(5) Ma, X.; Hahn, K.; Sanchez, S. J. Am. Chem. Soc. 2015, 137 (15), 4976-4979.

(6) Srivastava, S. K.; Medina-Sanchez, M.; Koch, B.; Schmidt, O. G. Adv. Mater. 2015, 832-837.

(7) Nelson, B. J.; Kaliakatsos, I. K.; Abbott, J. J. Annu. Rev. Biomed. Eng. 2010, 12, 5585 .

(8) Sacanna, S.; Korpics, M.; Rodriguez, K.; Colón-Meléndez, L.; Kim, S.-H.; Pine, D. J.; Yi, G.-R. Nat. Commun. 2013, 4, 1688.

(9) Li, J.; Gao, W.; Dong, R.; Pei, A.; Sattayasamitsathit, S.; Wang, J. Nat. Commun. 2014, 5, 5026.

(10) Srivastava, S. K.; Guix, M.; Schmidt, O. G. Nano Lett. 2015, 16, 817-821.

(11) Gao, W.; Wang, J. ACS Nano 2014, No. 4, 3170-3180.

(12) Soler, L.; Sánchez, S. Nanoscale 2014, 6 (13), 7175.

(13) Vilela, D.; Parmar, J.; Zeng, Y.; Zhao, Y.; Sanchez, S. Nano Lett. 2016, acs.nanolett.6b00768.

(14) Soler, L.; Magdanz, V.; Fomin, V. M.; Sanchez, S.; Schmidt, O. G. ACS Nano 2013, 7 (11), 9611-9620.

(15) Wang, H.; Pumera, M. Chem. Rev. 2015, 115 (16), 8704-8735.

(16) Sanchez, S.; Soler, L.; Katuri, J. Angew. Chemie - Int. Ed .2015, 54 (5), 1414-1444.

(17) Wang, Y.; Hernandez, R. M.; Bartlett, D. J.; Bingham, J. M.; Kline, T. R.; Sen, A.; Mallouk, T. E. Langmuir 2006, 22 (25), 10451-10456.

(18) Laocharoensuk, R.; Burdick, J.; Wang, J. ACS Nano 2008, 2 (5), 1069-1075.

(19) Paxton, W. F.; Kistler, K. C.; Olmeda, C. C.; Sen, A.; St Angelo, S. K.; Cao, Y.; Mallouk, T. E.; Lammert, P. E.; Crespi, V. H. J. Am. Chem. Soc. 2004, 126 (41), 13424-13431.

(20) Ota, S.; Wang, S.; Wang, Y.; Yin, X.; Zhang, X. Nano Lett. 2013, 13 (6), 2766-2770.

(21) Wu, J.; Balasubramanian, S.; Kagan, D.; Manesh, K. M.; Campuzano, S.; Wang, J. Nat. Commun. 2010, 1 (4), 1-6. 
(22) Calvo-Marzal, P.; Manesh, K. M.; Kagan, D.; Balasubramanian, S.; Cardona, M.; Flechsig, G.-U.; Posner, J.; Wang, J. Chem. Commun. 2009, No. 30, 4509-4511.

(23) Williams, B. J.; Anand, S. V; Rajagopalan, J.; Saif, M. T. A. Nat. Commun. 2014, 5, 3081 .

(24) Nawroth, J. C.; Lee, H.; Feinberg, A. W.; Ripplinger, C. M.; McCain, M. L.; Grosberg, A.; Dabiri, J. O.; Parker, K. K. Nat. Biotechnol. 2012, 30 (8), 792-797.

(25) Dreyfus, R.; Baudry, J.; Roper, M. L.; Fermigier, M.; Stone, H.; Bibette, J. Nature 2005, 437 (7060), 862-865.

(26) Ghosh, A.; Fischer, P. Nano Lett. 2009, 9 (6), 2243-2245.

(27) Snezhko, A.; Belkin, M.; Aranson, I.; Kwok, W.-K. Phys. Rev. Lett. 2009, 102 (11), 118103.

(28) Zhang, L.; Abbott, J. J.; Dong, L.; Peyer, K. E.; Kratochvil, B. E.; Zhang, H.; Bergeles, C.; Nelson, B. J. Nano Lett. 2009, 9 (10), 3663-3667.

(29) Sing, C. E.; Schmid, L.; Schneider, M. F.; Franke, T.; Alexander-Katz, A. Proc. Natl. Acad. Sci. U. S. A. 2010, 107 (2), 535-540.

(30) Jang, B.; Gutman, E.; Stucki, N.; Seitz, B. F.; Wendel-García, P. D.; Newton, T.;Pokki, J.; Ergeneman, O.; Pané, S.; Or, Y.; Nelson, B. J. Nano Lett . 2015, 15, 4829-4833.

(31) Tasci, T. O.; Herson, P. S.; Neeves, K. B.; Marr, D. W. M. Nat. Commun. 2016, 7, 10225 .

(32) Li, J.; Li, T.; Xu, T.; Kiristi, M.; Liu, W.; Wu, Z.; Wang, J. Nano Lett. 2015, 15 (7), 4814-4821.

(33) Gao, W.; Feng, X.; Pei, A.; Kane, C. R.; Tam, R.; Hennessy, C.; Wang, J. Nano Lett. 2014, 14 (1), 305-310.

(34) Loget, G.; Kuhn, A. Nat. Commun. 2011, 2, 535.

(35) Camacho-Lopez, M.; Finkelmann, H.; Palffy-Muhoray, P.; Shelley, M. Nat. Mater. 2004, 3 (5), 307-310.

(36) Jiang, H.-R.; Yoshinaga, N.; Sano, M. Phys. Rev. Lett. 2010, 105 (26), 268302.

(37) Maggi, C.; Saglimbeni, F.; Dipalo, M.; De Angelis, F.; Di Leonardo, R. Nat. Commun. 2015, 6,7855 .

(38) Feng, J.; Yuan, J.; Cho, S. K. Lab Chip 2015, 15, 1554-1562.

(39) Wang, W.; Castro, L. A.; Hoyos, M.; Mallouk, T. E. ACS Nano 2012, 6 (7), 61226132.

(40) Ahmed, D.; Lu, M.; Nourhani, A.; Lammert, P. E.; Stratton, Z.; Muddana, H. S.; Crespi, V. H.; Huang, T. J. Sci. Rep. 2015, 5, 9744.

(41) Dijkink, R. J.; Dennen, J. P. Van Der; Ohl, C. D.; Prosperetti, A. J. Micromech. 
Microeng. 2006, 16 (8), 1653-1659.

(42) Bertin, N.; Spelman, T. A.; Stephan, O.; Gredy, L.; Bouriau, M.; Lauga, E.; Marmottant, P. Phys. Rev. Appl. 2015, 4 (6), 064012.

(43) Parmar, J.; Ma, X.; Katuri, J.; Simmchen, J.; Stanton, M. M.; Trichet-Paredes, C.; Soler, L.; Sanchez, S. Sci. Technol. Adv. Mater.2015, 16 (1), 014802.

(44) Mirkovic, T.; Foo, M. L.; Arsenault, A. C.; Fournier-Bidoz, S.; Zacharia, N. S.; Ozin, G. A. Nat. Nanotechnol. 2007, 2 (9), 565-569.

(45) Leissa, A. W. J. Sound Vib. 1973, 31 (3), 257-293.

(46) Rossing, T. D. Am. J. Phys. 1982, 50 (3), 271.

(47) Dorrestijn, M.; Bietsch, A.; Acikalin, T.; Raman, A.; Hegner, M.; Meyer, E.; Gerber, C. Phys. Rev. Lett. 2007, 98 (2), 1-4.

(48) Leibacher, I.; Schatzer, S.; Dual, J. Lab Chip 2014, 14 (3), 463-470.

(49) Friend, J.; Yeo, L. Y. Rev. Mod. Phys. 2011, 83 (2), 647-704.

(50) Wiklund, M.; Green, R.; Ohlin, M. Lab Chip 2012, 12 (14), 2438-2451.

(51) Ahmed, D.; Chan, C. Y.; Lin, S.-C. S.; Muddana, H. S.; Nama, N.; Benkovic, S. J.; Huang, T. J. Lab Chip 2013, 13 (3), 328-331.

(52) Bruus, H. Lab Chip 2012, 12 (9), 1578-1586.

(53) Ahmed, D.; Muddana, H. S.; Lu, M.; French, J. B.; Ozcelik, A.; Fang, Y.; Butler, P. J.; Benkovic, S. J.; Manz, A.; Huang, T. J. Anal. Chem. 2014, 86, 11803-11810.

(54) Ahmed, D.; Ozcelik, A.; Bojanala, N.; Nama, N.; Upadhyay, A.; Chen, Y.; HannaRose, W.; Huang, T. J. Nat. Commun. 2016, 7, 11085.

(55) Hahn, P.; Leibacher, I.; Baasch, T.; Dual, J. Lab Chip 2015, 15, 4302-4313.

(56) Muller, P. B.; Rossi, M.; Marín, A. G.; Barnkob, R.; Augustsson, P.; Laurell, T.; Kähler, C. J.; Bruus, H. Phys. Rev. E 2013, 88 (2), 1-12.

(57) Schwarz, T.; Hahn, P.; Petit-Pierre, G.; Dual, J. Microfluid. Nanofluidics 2014, 18 (1), 65-79.

(58) Karlsen, J. T.; Bruus, H. Phys. Rev. E 2015, 92 (4), 043010.

(59) Martin, C. R.; Preedy, V. R.; Hunter, R. J. Nanomedicine and the Nervous System; CRC Press: Boca Raton, FL, 2012. 

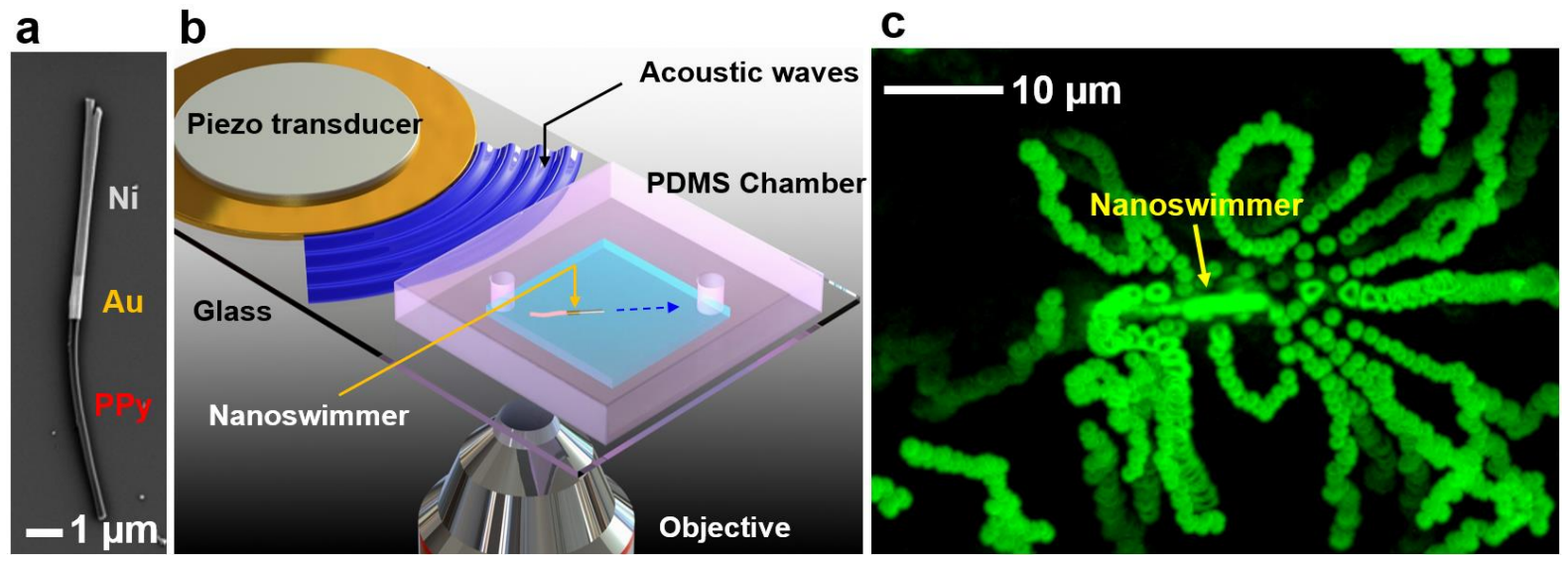

Figure 1. The design of the acoustic nanoswimmer and the experimental details. (a) Scanning electron microscope (SEM) image of a nanoswimmer, composed of a PPy tail and an $\mathrm{Au} / \mathrm{Ni}$ head. (b) Schematic of the experimental setup for traveling acoustic waves. A piezoelectric transducer, which is used to generate acoustic waves, is bonded adjacent to a PDMS based microchannel. The PDMS wall is made thick to minimize acoustic waves scattering/reflection from boundaries. (c) Acoustic oscillation of the nanoswimmer generates acoustic microstreaming (pseudo-colored green) in water (see also Supplementary Video 1). This is obtained by fixing the swimmer to the PDMS wall of the chamber. In the presence of ultrasound, the structure oscillates, which brings forth microstreaming in the surrounding liquid. This microstreaming effect is captured by introducing $1 \mu \mathrm{m}$ tracer particles (Bangs Laboratory), the image sequence is then superimposed for multiple images to see the trajectory of the vortices. 


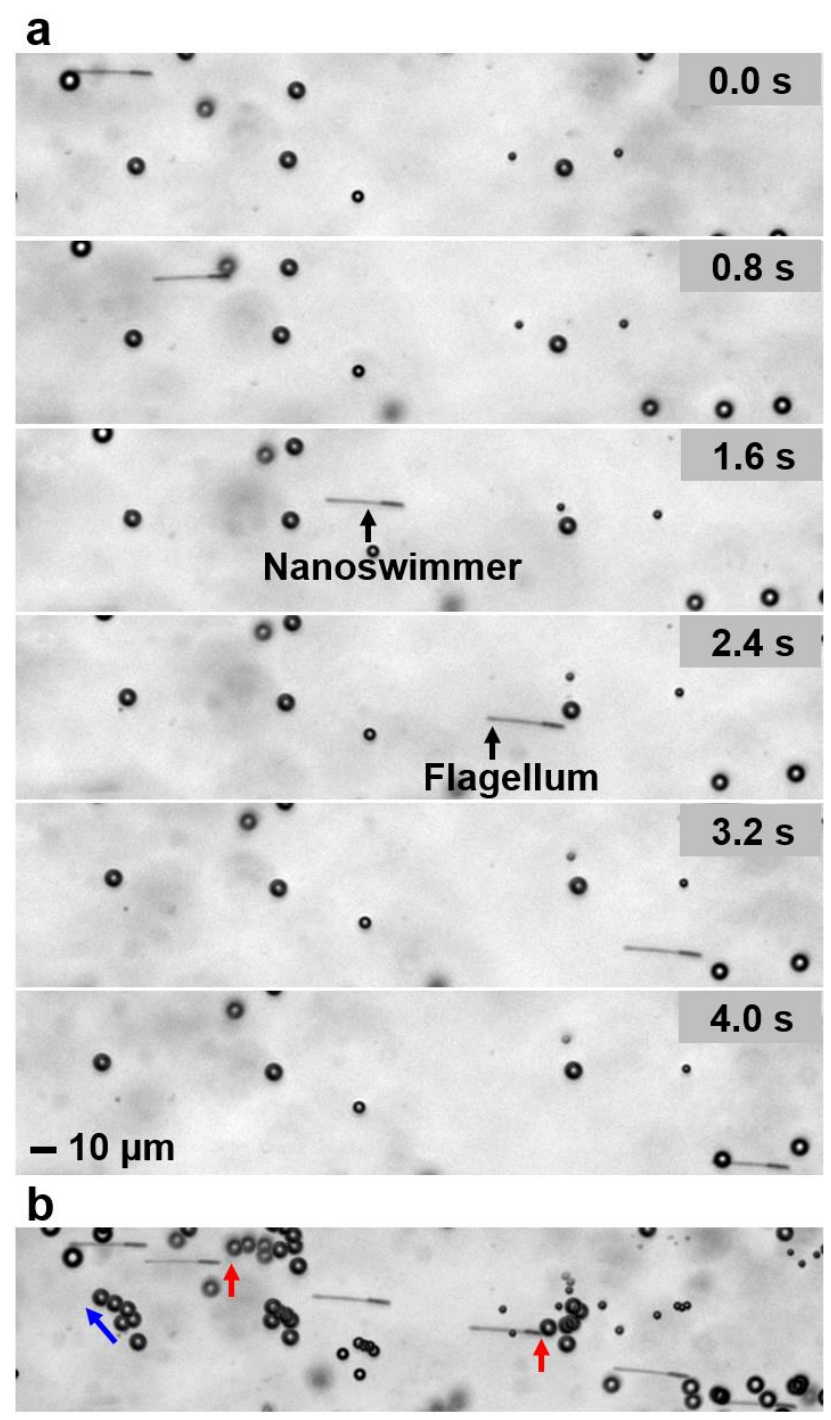

Figure 2. (a) Image sequence demonstrates translation motion of the acoustic nanoswimmer through water containing polystyrene particles (moving left to right) at $\sim 77 \mathrm{kHz}$ and $10 \mathrm{~V}_{\mathrm{PP}}$, respectively. The swimmer moving faster than the polystyrene particles suggest that the standing wave field does not predominantly drive the swimmer. (b) Superimposed images of (a) to show particle motion in the liquid in the presence of acoustic waves. Blue and red arrows demonstrate flow direction of the fluid and collision between a swimmer and a particle, respectively (see Supplementary Video 2). 

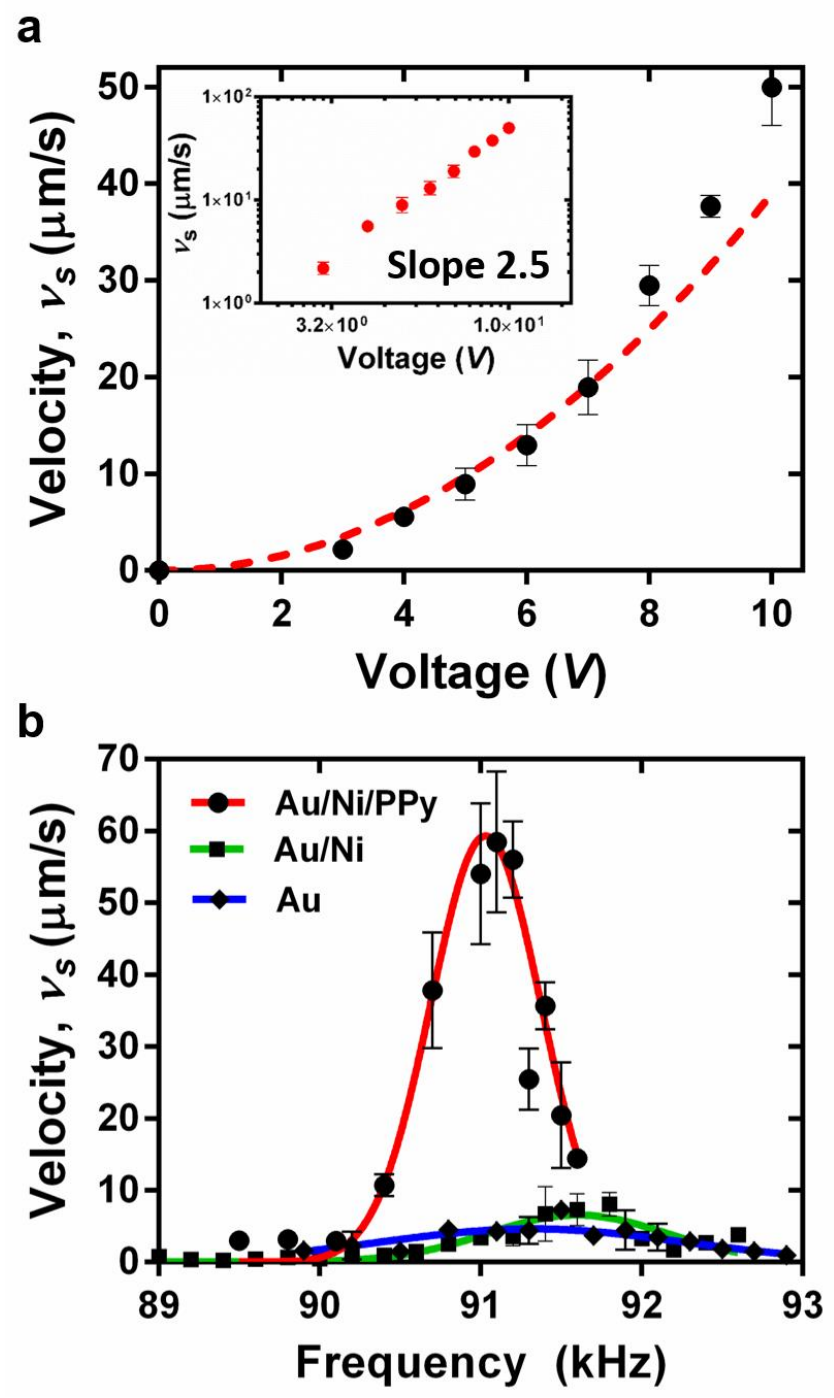

Figure 3. Velocity and frequency characterization of nanoswimmers. (a) The nanoswimmers immersed in water propel at a speed nearly proportional to the amplitude square of the driving voltage. (b) The frequency response of $\mathrm{Au} / \mathrm{Ni} / \mathrm{PPy}$ (nanoswimmer), $\mathrm{Au} / \mathrm{Ni}$ and $\mathrm{Au}$ particles, respectively. Fig. $3 \mathrm{~b}$ only compares the velocities of the swimmer and the metallic entities in the presence and the absence of the flexible tails. The important fact to note is that the propulsion velocities of the swimmers containing flexible tails have velocities an order of magnitude higher. The smaller peaks are not due to resonances of the structure but probably due to a transducer resonance. 


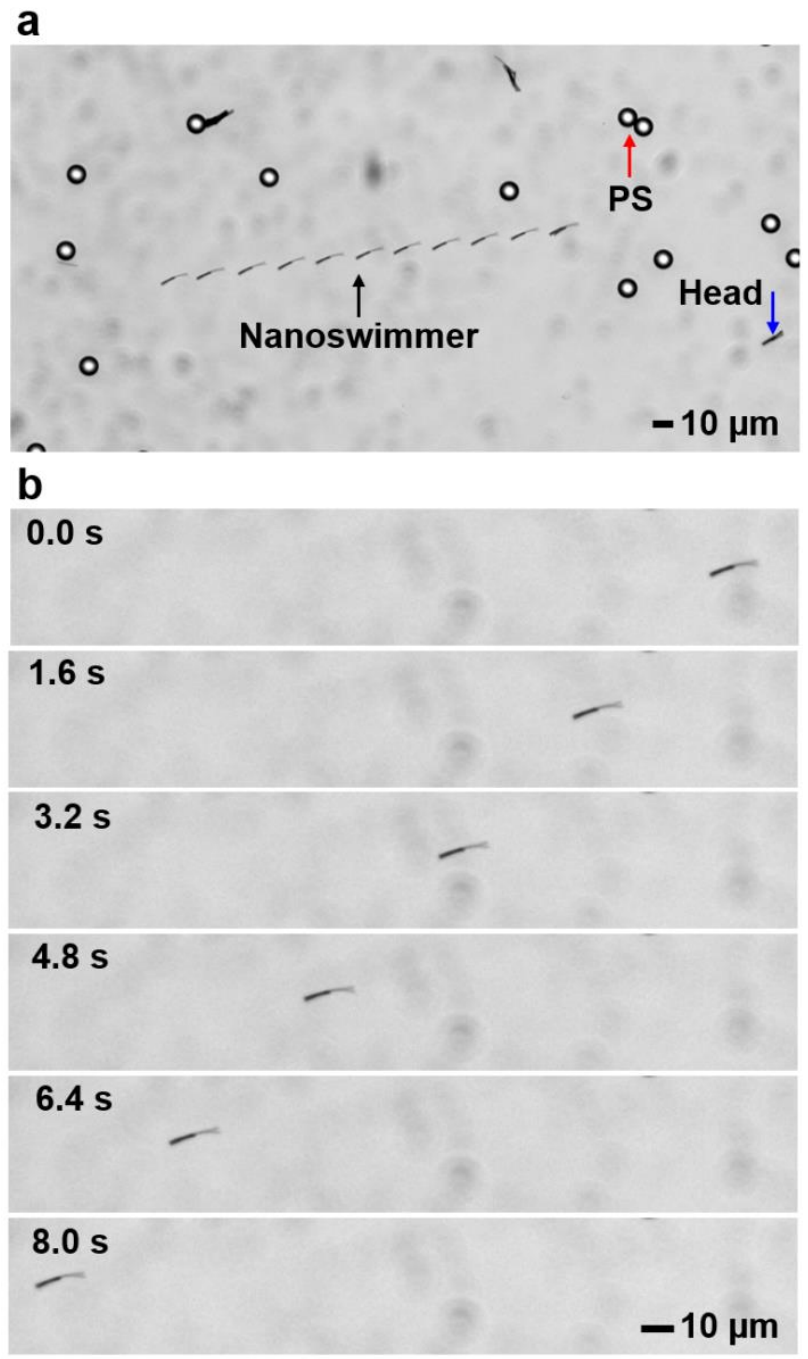

Figure 4. Propulsion of a nanoswimmer in a predominant traveling acoustic wave. (a) Superimposed images demonstrating propulsion (moving right to left) in water dispersed with $10 \mu \mathrm{m}$ polystyrene particles. Red and blue arrows mark the stationary particles and presumably the head of the swimmer, respectively (see also Supplementary Video 3). (b) Enlarged image sequence of (a) demonstrates propulsion at a uniform velocity (see also Supplementary Video 4). 

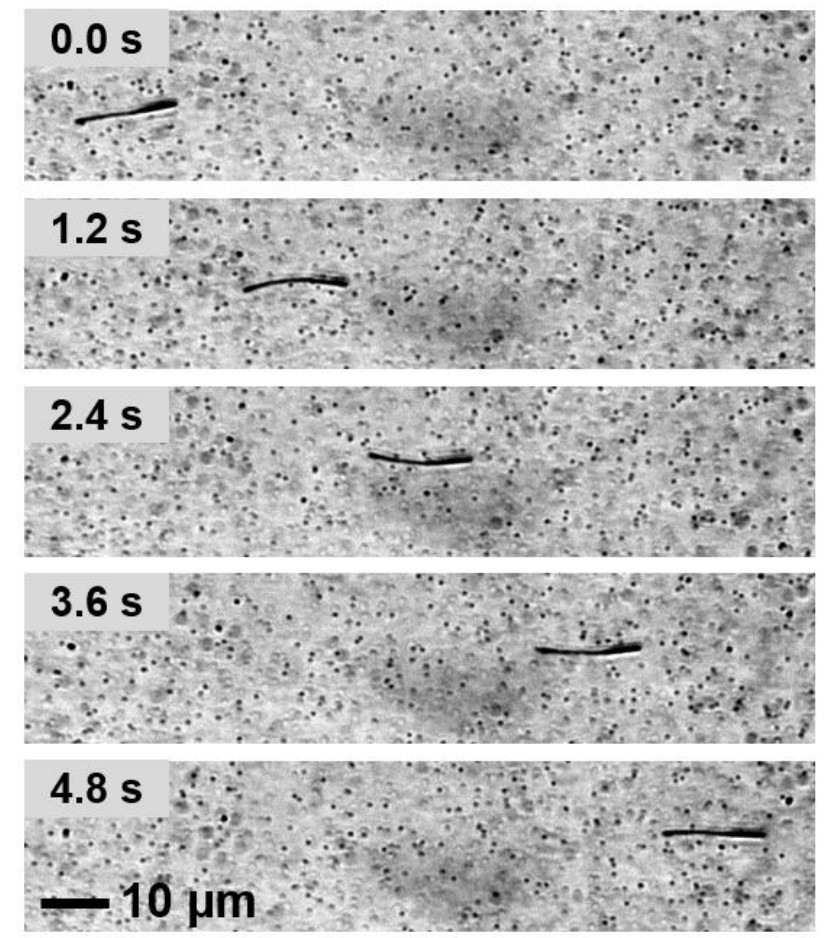

Figure 5. Images sequence captures the translational motion (moving left to right) of the nanoswimmer moving through water containing $0.5 \mu \mathrm{m}$ polystyrene particles (see also Supplementary Video 5). The swimmer moving faster than the polystyrene particles suggest that the external bulk streaming does not predominantly drive the swimmer. 

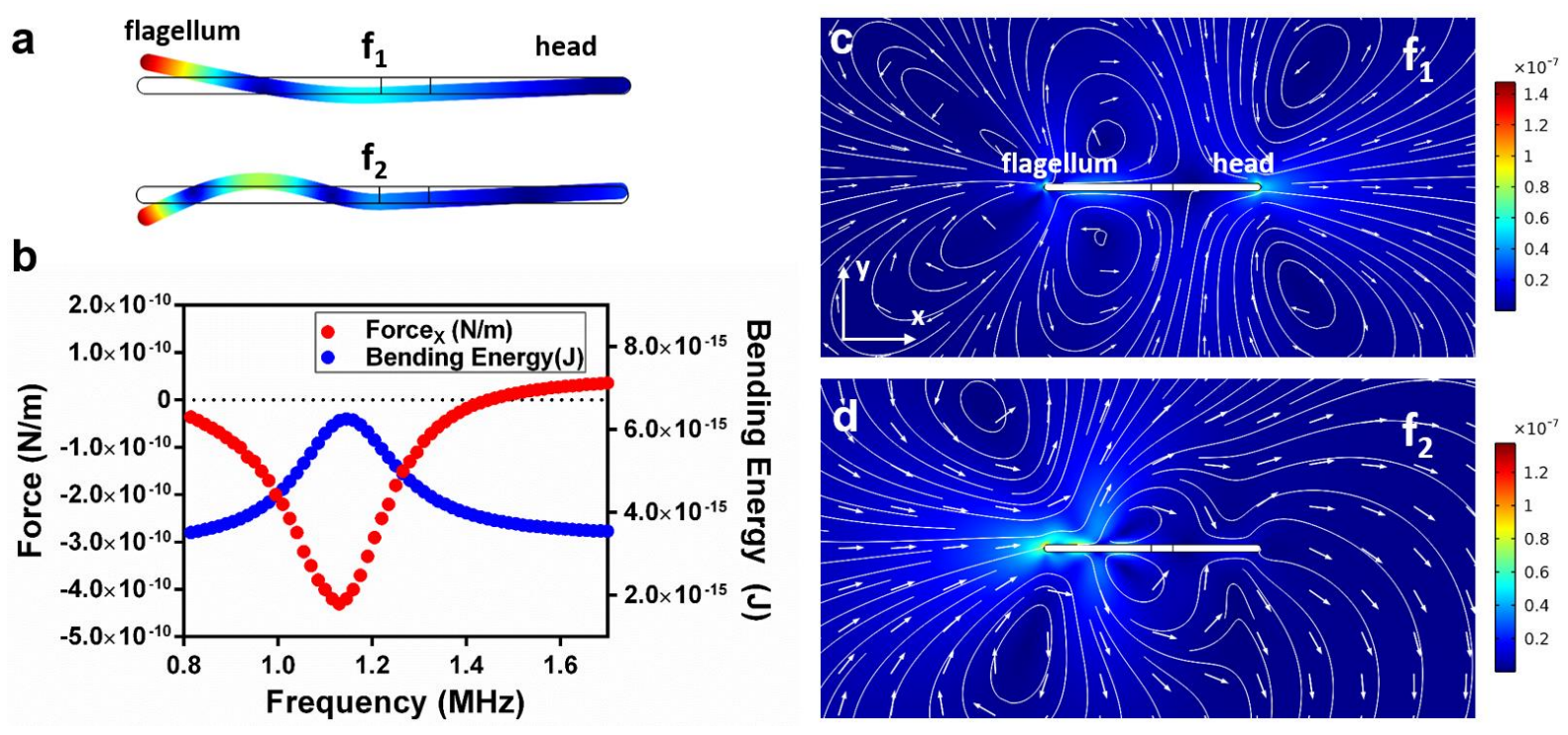

Figure 6. (a) Simulation results demonstrating the first $(240 \mathrm{kHz})$ and the second (1.13 MHz) eigenmodes of the nanoswimmer in water. The first and second harmonics were estimated using the first order simulations. We simulated multiple frequencies in our range of interest and the first order simulations were carried out for each frequency. (b) The plots of force and bending energy versus frequencies in $2 \mathrm{D}$. The simulation was carried to obtain the first and second order terms, which subsequently were used in combination with equation (8) to get the total force. The numerical results suggest that the swimmer excited at the second harmonics will move in the direction of the tail. However, we have not yet been able to verify this experimentally. The simulation at the first harmonics, although yielding only qualitative results, shows a resulting force in the direction of the swimmer's head as seen experimentally. We like to mention that the point of the numerical simulation is to show that the resonance of the swimmer can be excited by an external wave and leads to amplification of the acoustic forces. (c) The streamlines of $\mathbf{v}_{2}$ showing the microstreaming flow pattern of the nanoswimmer excited at (c) the first harmonic and (d) the second harmonic. 